\title{
Minimizing Intra-edge Crossings in Wiring Diagrams and Public Transportation Maps
}

\author{
Marc Benkert ${ }^{1, \star}$, Martin Nöllenburg ${ }^{1, \star}$, Takeaki Uno ${ }^{2}$, and Alexander Wolff $^{1, \star}$ \\ ${ }^{1}$ Department of Computer Science, Karlsruhe University, Germany \\ http://i11www.iti.uka.de/algo/group \\ ${ }^{2}$ National Institute of Informatics, Tokyo, Japan \\ uno@nii.jp
}

\begin{abstract}
In this paper we consider a new problem that occurs when drawing wiring diagrams or public transportation networks. Given an embedded graph $G=(V, E)$ (e.g., the streets served by a bus network) and a set $L$ of paths in $G$ (e.g., the bus lines), we want to draw the paths along the edges of $G$ such that they cross each other as few times as possible. For esthetic reasons we insist that the relative order of the paths that traverse a node does not change within the area occupied by that node.

Our main contribution is an algorithm that minimizes the number of crossings on a single edge $\{u, v\} \in E$ if we are given the order of the incoming and outgoing paths. The difficulty is deciding the order of the paths that terminate in $u$ or $v$ with respect to the fixed order of the paths that do not end there. Our algorithm uses dynamic programming and takes $O\left(n^{2}\right)$ time, where $n$ is the number of terminating paths.
\end{abstract}

\section{Introduction}

In wiring diagrams or public transportation networks many paths must be drawn on the same underlying graph, see Figures 1 and 2, In order to make the resulting layout as understandable as possible it is desirable to (a) avoid crossings wherever possible and (b) insist that the relative order of the lines that traverse a node does not change in that node. For example, note that the subway line 5, which passes under the main station of Cologne (Köln Hbf), crosses lines 16-19 south of the station in the clipping of the public transport map of Cologne in Figure 2. The crossing is not hidden under the rectangle that represents the station. This makes it easier to follow the subway lines visually.

We model the problem as follows. We assume that we are given an undirected connected graph $G=(V, E)$ together with an embedding in the plane. The graph represents the underlying structure of the wiring or the road/tracks in the case of a transportation network. We are also given a set $L$ of lines in $G$. They represent the cables in a wiring diagram or the lines in a transportation network. A line $\ell \in L$ is an edge sequence $e_{1}=\left\{v_{0}, v_{1}\right\}, e_{2}=\left\{v_{1}, v_{2}\right\}, \ldots, e_{k}=\left\{v_{k-1}, v_{k}\right\} \in E$

\footnotetext{
* Supported by grant WO 758/4-2 of the German Research Foundation (DFG).
} 


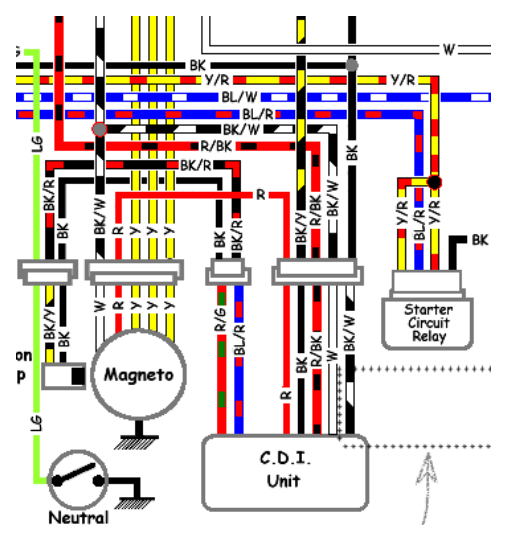

Fig. 1. Clipping of a wiring diagram

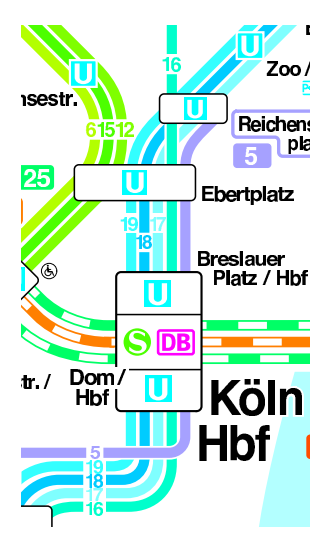

Fig. 2. Clipping of the public transport network of Cologne

that forms a simple path in $G$. The stations $v_{0}$ and $v_{k}$ are the terminal stations for line $\ell$ while $v_{1}, \ldots, v_{k-1}$ are intermediate stations. Our aim is to draw all lines in $L$ such that the number of crossings among pairs of lines in $L$ is minimized.

To get a flavor of the problem observe that the structure of $G$ enforces certain crossings, see Figure [3(a): lines $\ell_{1}$ and $\ell_{2}$ use exactly the path $\left\langle u, w_{1}, w_{2}, v\right\rangle$ together. The graph structure (indicated by the first and last line segments of each line) enforces that $\ell_{1}$ enters station $u$ above $\ell_{2}$ while it leaves $v$ below $\ell_{2}$, thus $\ell_{1}$ and $\ell_{2}$ have to cross somewhere between $u$ and $v$. However, fixing the location of the crossing of $\ell_{1}$ and $\ell_{2}$ determines crossings with other lines that have a terminal stop in $w_{1}$ or $w_{2}$. If there is a line $\ell$ that enters $u$ between $\ell_{1}$ and $\ell_{2}$ and terminates at $w_{2}$ (see Figure 3(b)), the crossing between $\ell_{1}$ and $\ell_{2}$ should be placed between $w_{2}$ and $v$. Now suppose there is another line $\ell^{\prime}$ that enters $v$ between $\ell_{2}$ and $\ell_{1}$ from the right and terminates at $w_{2}$, see Figure 3(c). Then at least one of the lines $\ell$ and $\ell^{\prime}$ intersects one of the lines $\ell_{1}$ or $\ell_{2}$, no matter where the crossing between $\ell_{1}$ and $\ell_{2}$ is placed.

We can abstract from geometry as follows. Given an edge $\{u, v\}$ in $G$ and the orders of the lines in $u$ and $v$ that traverse $u$ and $v$, respectively, we ask

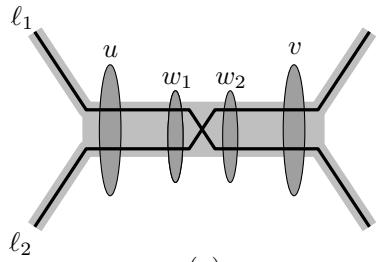

(a)

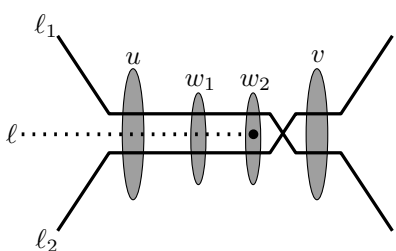

(b)

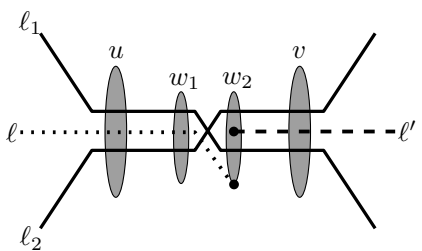

(c)

Fig. 3. Different placements of the necessary intersection between lines $\ell_{1}$ and $\ell_{2}$ on the path $u, w_{1}, w_{2}, v$. In (c) at least one of the lines $\ell$ and $\ell^{\prime}$ has to intersect one of the lines $\ell_{1}$ or $\ell_{2}$. 
for the order of all lines that enter $\{u, v\}$ in $u$ and for the order in which all lines leave $\{u, v\}$ in $v$. Then the number of crossings on $\{u, v\}$ is the number of transpositions needed to convert one order into the other. The difficulty is deciding the order of the lines that terminate in $u$ or $v$ with respect to the fixed order of the lines that traverse both $u$ and $v$. This is the one-edge layout problem that we study in Section 2 .

In contrast to the well-known NP-hard problem of minimizing crossings in a two-layer bipartite graph [3] the one-edge layout problem is polynomially solvable. The main reason is that there is an optimal layout of the lines that pass through edge $\{u, v\}$ such that no two lines that terminate in $u$ intersect and no two lines that terminate in $v$ intersect. This observation allows us to split the problem and to apply dynamic programming. It is then rather easy to come up with an $O\left(n^{5}\right)$-time solution and with some effort we could reduce the running time to $O\left(n^{2}\right)$, where $n$ is the number of lines that do not terminate in $u$ or $v$.

A solution of the general line layout problem, i.e., a simultaneous crossingminimal solution for all edges of a graph, would be of interest as a second (and mostly independent) step for drawing bus or metro maps, a topic that has received some attention lately, see the work of Nöllenburg and Wolff [4] and the references therein. However, in that direction of research the focus has so far been exclusively on drawing the underlying graph nicely, and not on how to embed the bus or metro lines along the network. We give some hints in Section 3 why already the two-edge layout problem seems to be substantially harder than the one-edge layout.

A vaguely similar problem has been considered by Cortese et al. [1. Given the drawing of a planar graph $G$ they widen the edges and vertices of the drawing and ask if a given combinatorial cycle in $G$ has a plane embedding in the widened drawing.

\section{A Dynamic Program for One-Edge Layout}

In this section we consider the following special case of the problem.

\section{Problem 1. One-edge layout}

We are given a graph $G=(V, E)$ and an edge $e=\{u, v\} \in E$. Let $L_{e}$ be the set of lines that use $e$. We split $L_{e}$ into three subgroups: $L_{u v}$ is the set of lines that pass through $u$ and $v$, i.e., neither $u$ or $v$ is a terminal station. $L_{u}$ is the set of lines that pass through $u$ and for which $v$ is a terminal station and $L_{v}$ is the set of lines that pass through $v$ and for which $u$ is a terminal station. We assume that there are no lines that exclusively use the edge $\{u, v\}$ as they could be placed top- or bottommost without causing any intersections. Furthermore we assume that the lines for which $u$ is an intermediate station, i.e., $L_{u v} \cup L_{u}$, enter $u$ in a predefined order $S_{u}$. Analogously, we assume that the lines for which $v$ is an intermediate station, i.e., $L_{u v} \cup L_{v}$, enter $v$ in a predefined order $S_{v}$. The task is to find a layout of the lines in $L_{e}$ such that the number of pairs of intersecting lines is minimized. 
Note that the number of crossings is determined by inserting the lines in $L_{u}$ into the order $S_{v}$ and by inserting the lines in $L_{v}$ into $S_{u}$. The task is to find insertion orders that minimize the number of crossings. Observe that the orders $S_{u}$ and $S_{v}$ themselves already determine the number of crossings between pairs of lines in $L_{u v}$ and that the insertion orders of $L_{u}$ in $S_{v}$ and of $L_{v}$ in $S_{u}$ do not

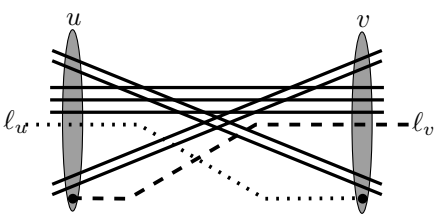

Fig. 4. The lines in $L_{u v}$ are drawn solid. In an optimal solution $\ell_{u} \in$ $L_{u}$ and $\ell_{v} \in L_{v}$ intersect. change this number. Thus, we will not take crossings between lines in $L_{u v}$ into account anymore. On the other hand, fixing an insertion order affects the number of crossings between lines in $L_{u} \cup L_{v}$ and $L_{u v}$ and the number of crossings between lines in $L_{u}$ and $L_{v}$ in a non-trivial way. Figure 4 shows that a line $\ell_{u} \in L_{u}$ can indeed cross a line $\ell_{v} \in L_{v}$ in the unique optimal solution. Throughout the paper lines in $L_{u v}$ are drawn solid, lines in $L_{u}$ dotted and lines in $L_{v}$ dashed. We will now show that no two lines in $L_{u}$ (and analogously in $L_{v}$ ) cross in an optimal solution. This nice property is the key for solving the one-edge layout in polynomial time.

Lemma 1. In any optimal solution for the one-edge layout problem no pair of lines in $L_{u}$ and no pair of lines in $L_{v}$ intersects.

Proof. Assume to the contrary that there is an optimal solution $\sigma$ with a pair of lines in $L_{u}$ that intersects. Among all such pairs in $\sigma$ let $\left\{\ell, \ell^{\prime}\right\}$ be the one whose intersection point $p$ is rightmost. W.l.o.g. $\ell$ is above $\ell^{\prime}$ in $u$. Let $\ell_{p}$ and $\ell_{p}^{\prime}$ be the parts of $\ell$ and $\ell^{\prime}$ to the right of $p$, see Figure 5(a). Since $\sigma$ is crossing minimal, the courses of $\ell_{p}$ and $\ell_{p}^{\prime}$ intersect the minimum number of lines in $L_{u v} \cup L_{v}$ in order to get from $p$ to $v$. In particular, the number of crossings between $\ell_{p}$ and lines of $L_{u v} \cup L_{v}$ and between $\ell_{p}^{\prime}$ and lines of $L_{u v} \cup L_{v}$ must be the same otherwise we could place $\ell_{p}$ parallel to $\ell_{p}^{\prime}$ (or vice versa) which would reduce the number of crossings. However, since the number of crossings to the right is the same we can easily get rid of the crossing between $\ell$ and $\ell^{\prime}$ by replacing $\ell_{p}$ by a copy of $\ell_{p}^{\prime}$ infinitesimally close above $\ell_{p}^{\prime}$, see Figure $5(\mathrm{~b})$. The proof for $L_{v}$ is analogous.

From now on we assume that no two lines of $L_{u}$ are consecutive in $S_{u}$ and analogously no two lines of $L_{v}$ are consecutive in $S_{v}$. The reason for this assumption is that a set of consecutive lines can simply be drawn parallelly in an optimal layout. Thus a single line suffices to determine the optimal course for the whole bundle. Technically, we can deal with this case by merging a bundle of $k$ consecutive lines of $L_{u}$ or $L_{v}$ to one line and assigning a weight of $k$ to it. The dynamic program will then run in a weighted fashion that counts $k \cdot k^{\prime}$ crossings for a crossing of two lines with weights $k$ and $k^{\prime}$. For simplification we only explain the unweighted version of the problem in detail. 


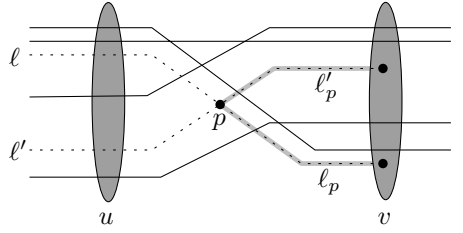

(a)

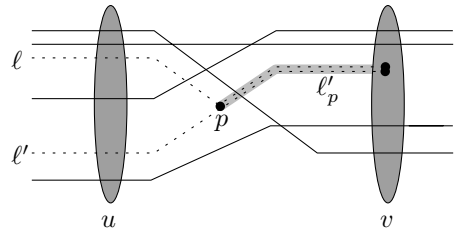

(b)

Fig. 5. Two lines of $L_{u}$ do not intersect in an optimal solution

Let $n, n_{u}$ and $n_{v}$ be the number of lines in $L_{u v}, L_{u}$ and $L_{v}$, respectively. Note that by the above assumption $n_{u}, n_{v} \leq n+1$ holds.

Recall that by assumption the lines in $L_{u v} \cup L_{u}$ enter $u$ in a predefined order and the lines in $L_{u v} \cup L_{v}$ leave $v$ in a predefined order. Let $S_{u}=\left(s_{1}^{u}<\cdots<\right.$ $\left.s_{n+n_{u}}^{u}\right)$ be the bottom-up order of lines in $L_{u v} \cup L_{u}$ in $u$ and $S_{v}=\left(s_{1}^{v}<\cdots<\right.$ $\left.s_{n+n_{v}}^{v}\right)$ be the bottom-up order of lines in $L_{u v} \cup L_{v}$ in $v$. A line $\ell$ in $L_{v}$ can terminate below $s_{1}^{u}$, between two neighboring lines $s_{i}^{u}, s_{i+1}^{u}$, or above $s_{n+n_{u}}^{u}$. We denote the position of $\ell$ by the index of the lower line and by 0 if it is below $s_{1}^{u}$. Let $S_{v} \mid L_{v}=\left(s_{\pi(1)}^{v}<\ldots<s_{\pi\left(n_{v}\right)}^{v}\right)$ denote the order on $L_{v}$ induced by $S_{v}$ and let $S_{u} \mid L_{u}=\left(s_{\mu(1)}^{u}<\ldots<s_{\mu\left(n_{u}\right)}^{u}\right)$ denote the order on $L_{u}$ induced by $S_{u}$. Here, $\pi$ and $\mu$ are injective functions that filter the lines $L_{v}$ out of all ordered lines $L_{u v} \cup L_{v}$ in $S_{v}$ and the lines $L_{u}$ out of all ordered lines $L_{u v} \cup L_{u}$ in $S_{u}$, see Figure 6.

Preprocessing. The orders $S_{u}$ and $S_{v}$ already determine the number of necessary crossings between pairs of lines in $L_{u v}$. Let $\mathrm{cr}_{u v}$ denote this number. Since $\mathrm{cr}_{u v}$ is fixed there is no need to consider the corresponding crossings in the minimization. We will now fix the course of a line in $L_{v}$. This line, say $\ell=s_{\pi(j)}^{v}$, has index $\pi(j)$ in $S_{v}$, and we fix the course of $\ell$ by choosing its terminal position $i$ in the order $S_{u}$. We denote the number of crossings between $\ell$ and all lines in $L_{u v}$ by $\operatorname{cr}_{v}(i, j)$. This number is determined as follows. The line $\ell$ crosses a line $\ell^{\prime} \in L_{u v}$ with left index $i^{\prime}$ and right index $j^{\prime}$ if and only if either it holds that $i^{\prime} \leq i$ and $j^{\prime}>\pi(j)$ or it holds that $i^{\prime}>i$ and $j^{\prime}<\pi(j)$. The table $\mathrm{cr}_{v}$ for all lines in $L_{v}$ has $\left(n+n_{u}+1\right) \times n_{v}=O\left(n^{2}\right)$ entries. For fixed $i$ we can compute the row $\operatorname{cr}_{v}(i, \cdot)$ as follows. We start with $j=1$ and compute the number of lines in $L_{u v}$ that intersect line $\ell$ with indices $i$ and $\pi(j)$. Then, we increment $j$ and obtain $\mathrm{cr}_{v}(i, j+1)$ by $\mathrm{cr}_{v}(i, j)$ minus the number of lines in $L_{u v}$ that are no longer intersected plus the lines that are newly intersected. As any of the $n$ lines in $L_{u v}$ receives this status 'no longer' or 'newly' at most once and this status can easily be checked by looking at $S_{v}$, this takes $O(n)$ time per row. Thus, in total we can compute the matrix $\mathrm{cr}_{v}$ in $O\left(n^{2}\right)$ time.

We define $\operatorname{cr}_{u}(i, j)$ analogously to be the number of crossings of the lines in $L_{u v}$ with a line in $L_{u}$ that has index $\mu(i)$ in $S_{u}$ and position $j$ in $S_{v}$. Computing $\mathrm{Cr}_{u}$ is analogous to $\mathrm{cr}_{v}$. 


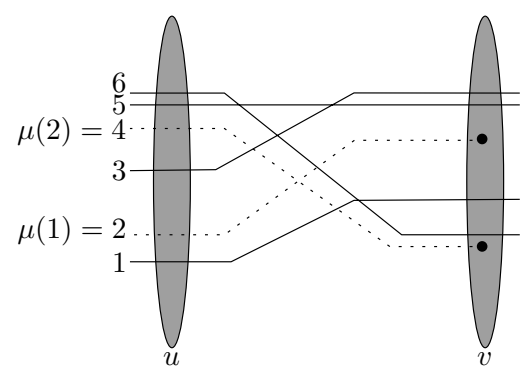

Fig. 6. The order $S_{u}$ and the induced suborder $S_{u} \mid L_{u}=\left(s_{2}^{u}<s_{4}^{u}\right)$

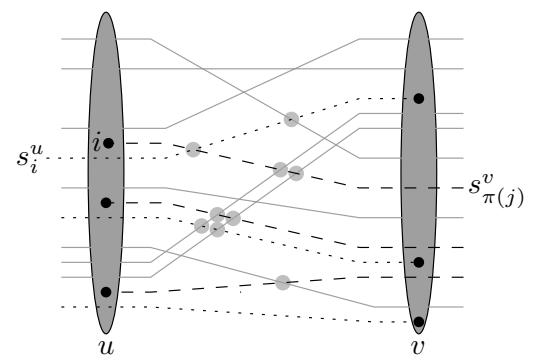

Fig. 7. Configuration corresponding to $F(i, j)$ : line $s_{\pi(j)}^{v}$ terminates at position $i$ in $u$

Dynamic Program. Assume that we fix the destination of $s_{\pi(j)}^{v}$ to some $i \in$ $\left\{0, \ldots, n+n_{u}\right\}$. Then we define $F(i, j)$ as the minimum number of crossings of (a) the lines in $\left\{s_{1}^{u}, \ldots, s_{i}^{u}\right\} \cap L_{u}$ with the lines in $L_{u v} \cup L_{v}$ and (b) the lines in $\left\{s_{1}^{v}, \ldots, s_{\pi(j)}^{v}\right\} \cap L_{v}$ with the lines in $L_{u v} \cup L_{u}$. This situation is depicted in Figure [7, where only the crossings indicated by gray disks are counted in $F(i, j)$. Then the values $F(i, j)$ define an $\left(n+n_{u}+1\right) \times n_{v}$-matrix $F$.

Once the last column $F\left(\cdot, n_{v}\right)$ of the matrix $F$ is computed, i.e., all lines in $L_{v}$ are placed, we can determine the optimal solution for $L_{e}$ as

$$
F^{*}:=\min \left\{F\left(i, n_{v}\right)+C\left(i, n+n_{u}, n_{v}+1\right) \mid i=0, \ldots, n\right\},
$$

where $C\left(i, n+n_{u}, n_{v}+1\right)$ is the remaining number of crossings of lines in $L_{u} \cap$ $\left\{s_{i+1}^{u}, \ldots, s_{n+n_{u}}^{u}\right\}$ with $L_{u v} \cup L_{v}$, which are not yet counted in $F\left(i, n_{v}\right)$.

Before turning to the recursive computation of $F(i, j)$ we introduce another notation. Let us assume that $s_{\pi(j-1)}^{v}$ terminates at position $k$ and $s_{\pi(j)}^{v}$ terminates at position $i$, where $0 \leq k \leq i \leq n+n_{u}$ and $j \in\left\{1, \ldots, n_{v}\right\}$. Then let $C(k, i, j)$ denote the minimum number of crossings that the lines $L_{k, i}^{u}:=$ $\left\{s_{k+1}^{u}, \ldots, s_{i}^{u}\right\} \cap L_{u}$ cause with $L_{u v} \cup L_{v}$. In other words $C(k, i, j)$ counts the minimal number of crossings of all lines of $L_{u}$ in the interval defined by the endpoints of the two lines $s_{\pi(j-1)}^{v}$ and $s_{\pi(j)}^{v}$. This situation is illustrated in Figure 8. where those crossings that are marked with gray disks are counted in the term $C(k, i, j)$. The following theorem gives the recursion for $F$ and shows its correctness.

Theorem 1. The values $F(i, j), i=0, \ldots, n+n_{u}, j=1, \ldots, n_{v}$, can be computed recursively by

$$
F(i, j)= \begin{cases}\min _{k \leq i}\left\{F(k, j-1)+C(k, i, j)+c r_{v}(i, j)\right\} & \text { if } i \geq 1, j \geq 2 \\ \sum_{l=1}^{j} c r_{v}(0, l) & \text { if } i=0, j \geq 1 \\ C(0, i, 1)+c r_{v}(i, 1) & \text { if } i \geq 1, j=1\end{cases}
$$

Proof. The base cases of Equation (1) consist of two parts. In the first row, an entry $F(0, j)$ means that all lines $s_{\pi(1)}^{v}, \ldots, s_{\pi(j)}^{v}$ terminate at position 0 in $u$ and 
hence the required number of crossings is just the number of crossings of these lines with $L_{u v}$, which equals the sum given in Equation (11). In the first column, an entry $F(i, 1)$ reflects the situation that line $s_{\pi(1)}^{v}$ terminates at position $i$. The required number of crossings in this case is $\operatorname{simply} \operatorname{cr}_{v}(i, 1)$, the number of crossings of $s_{\pi(1)}^{v}$ with $L_{u v}$, plus $C(0, i, 1)$, the number of crossings of $L_{0, i}^{u}$ with $L_{u v} \cup L_{v}$.

The general case of Equation (1) means that the value $F(i, j)$ can be composed of the optimal placement $F(k, j-1)$ of the lines below and including $s_{\pi(j-1)}^{v}$ (which itself terminates at some position $k$ below $i$ ), the number $C(k, i, j)$ of crossings of lines in $L_{u}$ in the interval between $k$ and $i$, and the number of crossings $\operatorname{cr}_{v}(i, j)$ of $s_{\pi(j)}^{v}$ at position $i$.

Due to Lemma 1 we know that $s_{\pi(j)}^{v}$ cannot terminate below $s_{\pi(j-1)}^{v}$ in an optimal solution. Hence, for $s_{\pi(j)}^{v}$ terminating at position $i$, we know that $s_{\pi(j-1)}^{v}$ terminates at some position $k \leq i$. For each $k$ we know by the induction hypothesis that $F(k, j-1)$ is the correct minimum number of crossings as defined above. In order to extend the configuration corresponding to $F(k, j-1)$ with the next line $s_{\pi(j)}^{v}$ in $L_{v}$ we need to add two terms: (a) the number of crossings of $L_{k, i}^{u}$ with $L_{u v} \cup L_{v}$, which is exactly $C(k, i, j)$, and (b) the number $\operatorname{cr}_{v}(i, j)$ of crossings that the line $s_{\pi(j)}^{v}$ (terminating at position $i$ ) has with $L_{u v}$. Note that potential crossings of $s_{\pi(j)}^{v}$ with lines in $L_{k, i}^{u}$ are already considered in the term $C(k, i, j)$. Figure 8 illustrates this recursion: $s_{\pi(j)}^{v}$ is placed at position $i$ in the order $S_{u}$, and $s_{\pi(j-1)}^{v}$ terminates at position $k$. The crossings of the configuration corresponding to $F(i, j)$ that are not counted in $F(k, j-1)$, are the $C(k, i, j)$ crossings of the marked, dotted lines of $L_{u}$ (indicated by gray disks) and the $\operatorname{cr}_{v}(i, j)$ encircled ones of $s_{\pi(j)}^{v}$ with $L_{u v}$.

Finally, we have to show that taking the minimum value of the sum in Equation (1) for all possible terminal positions $k$ of line $s_{\pi(j-1)}^{v}$ yields an optimal solution for $F(i, j)$. Assume to the contrary that there is a better solution $F^{\prime}(i, j)$. This solution induces a solution $F^{\prime}(k, j-1)$, where $k$ is the position of $s_{\pi(j-1)}^{v}$ in $S_{u}$. Lemma 1 restricts $k \leq i$ and hence $s_{\pi(j-1)}^{v}$ runs completely below $s_{\pi(j)}^{v}$. Therefore we have $F^{\prime}(k, j-1) \leq F^{\prime}(i, j)-C(k, i, j)-\operatorname{cr}_{v}(i, j)<$ $F(i, j)-C(k, i, j)-\mathrm{cr}_{v}(i, j) \leq F(k, j-1)$. This contradicts the minimality of $F(k, j-1)$.

If we store in each cell $F(i, j)$ a pointer to the corresponding predecessor cell $F(k, j-1)$ that minimizes Equation (1) we can reconstruct the optimal edge layout: starting at the cell $F\left(i, n_{v}\right)$ that minimizes $F^{*}$, we can reconstruct the genesis of the optimal solution using backtracking. Obviously, using the combinatorial solution to place all endpoints of $L_{e}$ in the correct order and then connecting them with straight-line segments results in a layout that has exactly $F^{*}$ crossings in addition to $\mathrm{cr}_{u v}$, the invariable number of crossings of $L_{u v}$.

Now, we can give a first, naive approach: As mentioned earlier the tables $\mathrm{cr}_{u}$ and $\mathrm{cr}_{v}$ can be computed in $O\left(n^{2}\right)$ time. For the computation of one cell entry $C(k, i, j)$ we only have to look at the at most $n$ lines $L_{k, i}^{u}$ and their possible $n+1$ 


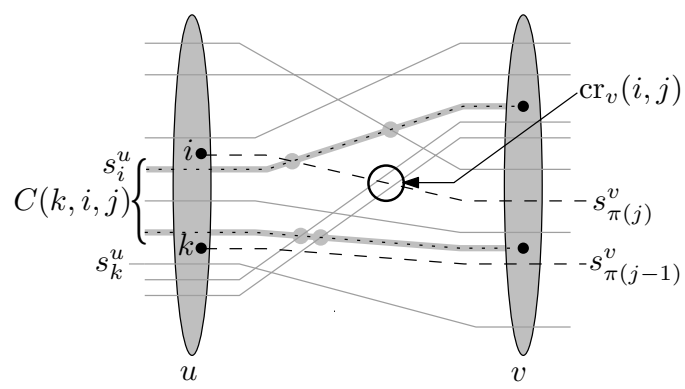

Fig. 8. The recursion for $F(i, j)$ : lines $s_{\pi(j-1)}^{v}$ and $s_{\pi(j)}^{v}$ terminate at pos. $k$ and $i$, resp. $C(k, i, j)=\min$. \# crossings of the marked dotted lines $L_{k, i}^{u}$ with $L_{u v} \cup L_{v}={ }^{\text {here }} 4$ $\operatorname{cr}_{v}(i, j)=$ number of crossings of $s_{\pi(j)}^{v}$ with $L_{u v} \quad$ here 2

terminal positions in $v$. Once we have fixed a terminal position of a line $\ell \in L_{k, i}^{u}$, we have to compute the number of crossings that $\ell$ has with $L_{u v} \cup L_{v}$. For the crossings with $L_{u v}$ we simply have to look at the corresponding value of $\mathrm{cr}_{u}$. For the crossings with $L_{v}$ it is sufficient to look at the index of the terminal position because we know that position $k$ is the terminal position of $s_{\pi(j-1)}^{v}$ and position $i$ is the terminal position of $s_{\pi(j)}^{v}$. Thus, computing one of the $O\left(n^{3}\right)$ cells of the table $C$ requires $O\left(n^{2}\right)$ time, so in total we need $O\left(n^{5}\right)$ time for filling $C$. This dominates the computation of the table $F$. In the remainder of this section we show how to speed up the computation of $F$ and $C$.

Improving the Running Time. Let us-for the moment-assume that the values $C(k, i, j)$ and $\operatorname{cr}_{v}(i, j)$ are available in constant time. Then the computation of the $\left(n+n_{u}+1\right) \times n_{v}$ matrix $F$ still needs $O\left(n^{3}\right)$ time because the minimum in Equation (1) is over a set of $O(n)$ elements. The following series of lemmas shows how we could bring the running time down to $O\left(n^{2}\right)$. First we show a relation for the entries of the matrix $C$.

Lemma 2. $C(k, i, j)$ is additive in the sense that $C(k, i, j)=C(k, l, j)+C(l, i, j)$ for $k \leq l \leq i$.

Proof. Since $C(k, i, j)$ denotes the number of crossings of the lines in $L_{u} \cap$ $\left\{s_{k+1}^{u}, \ldots, s_{i}^{u}\right\}$ and no two of these lines intersect each other (recall Lemma 1) we can split the layout corresponding to $C(k, i, j)$ at any position $l, k \leq l \leq i$ and get two (possibly non-optimal) configurations for the induced subproblems. This implies $C(k, i, j) \geq C(k, l, j)+C(l, i, j)$.

Conversely, we can get a configuration for $C(k, i, j)$ by putting together the optimal solutions of the subproblems. W.l.o.g. this introduces no additional crossings (they could be removed as in the proof of Lemma 1). Hence we have $C(k, i, j) \leq C(k, l, j)+C(l, i, j)$.

Now we show that we do not need to compute all entries of $C$. 
Lemma 3. Given the matrix $C$, the matrix $F$ can be computed in $O\left(n^{2}\right)$ time.

Proof. Having computed entry $F(i-1, j)$ we can compute $F(i, j)$ in constant time as follows:

$$
F(i, j)=\min \left\{\begin{array}{l}
F(i-1, j)+C(i-1, i, j)-\operatorname{cr}_{v}(i-1, j)+\operatorname{cr}_{v}(i, j), \\
F(i, j-1)+\mathrm{cr}_{v}(i, j)
\end{array}\right.
$$

The correctness follows from Equation (1), Lemma 2, and the fact that $C(i, i, j)$ vanishes:

$$
\begin{aligned}
& F(i, j) \stackrel{11}{=} \min \left\{\begin{array}{l}
\min _{k<i}\left\{F(k, j-1)+C(k, i, j)+\operatorname{cr}_{v}(i, j)\right\} \\
F(i, j-1)+C(i, i, j)+\operatorname{cr}_{v}(i, j)
\end{array}\right. \\
& \stackrel{\text { L. 2. }}{=} \min \left\{\begin{array}{l}
\min _{k \leq i-1}\left\{F(k, j-1)+C(k, i-1, j)+C(i-1, i, j)+\mathrm{cr}_{v}(i, j)\right\}, \\
F(i, j-1)+\operatorname{cr}_{v}(i, j)
\end{array}\right. \\
& \stackrel{11}{=} \min \left\{\begin{array}{l}
F(i-1, j)-\mathrm{cr}_{v}(i-1, j)+C(i-1, i, j)+\mathrm{cr}_{v}(i, j), \\
F(i, j-1)+\mathrm{cr}_{v}(i, j)
\end{array}\right.
\end{aligned}
$$

In the first column, we can reformulate the recursion for $i \geq 1$ as follows:

$$
\begin{aligned}
& F(i, 1) \stackrel{11}{=} C(0, i, 1)+\operatorname{cr}_{v}(i, 1) \\
& \stackrel{\text { LemmaL }}{=} C(0, i-1,1)+C(i-1, i, 1)+\operatorname{cr}_{v}(i, 1) \\
& \stackrel{11}{=} F(i-1,1)-\mathrm{cr}_{v}(i-1,1)+C(i-1, i, 1)+\mathrm{cr}_{v}(i, 1)
\end{aligned}
$$

Hence the whole matrix $F$ can be computed in $O\left(\left[n+n_{u}\right] \cdot n_{v}\right)=O\left(n^{2}\right)$ time.

Observe that due to the reformulation in Lemma 3 we only need the values $C(i-1, i, j)$ explicitly in order to compute $F$. Now we will show that we can compute these relevant values in $O\left(n^{2}\right)$ time. For simplification we introduce the following notation: $C^{\prime}(i, j):=C(i-1, i, j)$.

Lemma 4. The values $C^{\prime}(i, j)\left(i=1, \ldots, n+n_{u}, j=1, \ldots, n_{v}\right)$ can be computed in $O\left(n^{2}\right)$ time.

Proof. We compute $C^{\prime}(i, j)$ row-wise, i.e., we fix $i$ and increase $j$. Recall that $C(k, i, j)$ was defined as the minimal number of crossings of the lines in $L_{k, i}^{u}=$ $\left\{s_{k+1}^{u}, \ldots, s_{i}^{u}\right\} \cap L_{u}$ with the lines in $L_{u v} \cup L_{v}$ under the condition that $s_{\pi(j-1)}^{v}$ ends at position $k$ and $s_{\pi(j)}^{v}$ ends at position $i$. For $C^{\prime}(i, j)=C(i-1, i, j)$ this means we have to consider crossings of the set $L_{i-1, i}^{u}=\left\{s_{i}^{u}\right\} \cap L_{u}$. Hence, we distinguish two cases: either $s_{i}^{u}$ is a line in $L_{u v}$ and then $L_{i-1, i}^{u}$ is empty or $s_{i}^{u} \in L_{u}$ and we have to place the line $s_{i}^{u}$ optimally. Clearly, in the first case we have $C^{\prime}(i, j)=0$ for all $j$ as there is no line to place in $L_{i-1, i}^{u}$.

Now we consider the case that $s_{i}^{u} \in L_{u}$. For each $j$ we split the set of candidate terminal positions for $s_{i}^{u}$ into the intervals $[0, \pi(j-1)),[\pi(j-1), \pi(j))$, and $\left[\pi(j), n+n_{v}\right]$. Let $\operatorname{LM}(i, j), \operatorname{MM}(i, j)$, and $\operatorname{UM}(i, j)$ denote the minimum number of crossings of $s_{i}^{u}$ with $L_{u v} \cup L_{v}$ for terminal positions in $[0, \pi(j-$ $1)),[\pi(j-1), \pi(j))$, and $\left[\pi(j), n+n_{v}\right]$, respectively. Now we have $C^{\prime}(i, j)=$ 


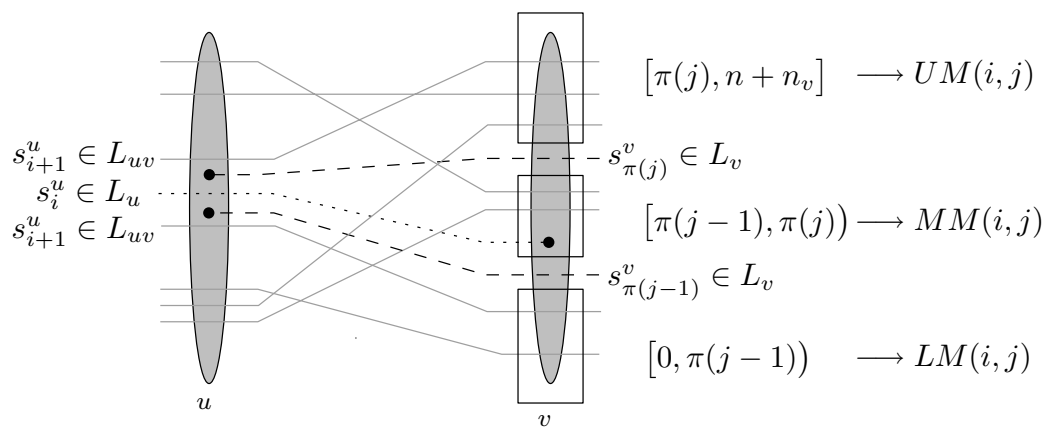

Fig. 9. Splitting the candidate terminal positions for $s_{i}^{u}$ into three intervals w.r.t. $\pi(j-1)$ and $\pi(j)$

$\min \{\operatorname{LM}(i, j), \operatorname{MM}(i, j), \operatorname{UM}(i, j)\}$ as the minimum of the three distinct cases. The situation is illustrated in Figure 9.

Next, we have to show how to compute MM, LM, and UM. First, we consider MM. Recall that $\mathrm{cr}_{u}(i, j)$ was defined as the number of crossings of the lines in $L_{u v}$ with the line $s_{\mu(i)}^{u}$ that terminates at position $j$ in $v$. It follows that

$$
\operatorname{MM}(i, j)=\min \left\{\operatorname{cr}_{u}\left(\mu^{-1}(i), k\right) \mid \pi(j-1) \leq k<\pi(j)\right\},
$$

where $\pi(0)$ is defined as 0 . Because of Lemma 1 there are no lines of $L_{v}$ intersecting the tunnel between $s_{\pi(j-1)}^{v}$ and $s_{\pi(j)}^{v}$. Hence, if the line $s_{i}^{u}$ terminates at position $k \in[\pi(j-1), \pi(j))$ it does not cross any line of $L_{v}$ and Equation (3) is correct. We can calculate $\operatorname{MM}(i, j)$ by a straight-forward minimum computation through $j=1, \ldots, n_{v}$ which takes $O\left(n+n_{v}\right)=O(n)$ time for each value of $i$.

Secondly, we consider LM. Initially, in the case that $j=1$ there is no line $s_{\pi(j-1)}^{v}$ and the corresponding interval is empty. Hence we set $\operatorname{LM}(i, 1)=\infty$. Then we recursively compute

$$
\operatorname{LM}(i, j+1)=\min \{\operatorname{LM}(i, j)+1, \operatorname{MM}(i, j)+1\} .
$$

Observe that for $\operatorname{LM}(i, j+1)$ we merge the previous intervals corresponding to $\operatorname{LM}(i, j)$ and $\operatorname{MM}(i, j)$. Moreover the line $s_{\pi(j)}^{v}$, which previously ended at position $i$, now terminates at position $i-1$. Hence, in order to reach its terminal position in the interval $[0, \pi(j))$, the line $s_{i}^{u}$ has to cross $s_{\pi(j)}^{v}$ in addition to the crossings counted before by $\operatorname{MM}(i, j)$ and $\operatorname{LM}(i, j)$. This explains the recursion in Equation (4). The computation again requires $O(n)$ time for each value of $i$.

Finally, we initialize $\operatorname{UM}\left(i, n_{v}\right)=1+\min \left\{\operatorname{cr}_{u}\left(\mu^{-1}(i), k\right) \mid \pi\left(n_{v}\right) \leq k \leq n+n_{v}\right\}$ as for $j=n_{v}$ the line $s_{i}^{u}$ crosses the line $s_{\pi\left(n_{v}\right)}^{v}$ but no other line of $L_{v}$. In decreasing order we compute

$$
\mathrm{UM}(i, j-1)=\min \{\mathrm{UM}(i, j)+1, \operatorname{MM}(i, j)+1\}
$$

analogously to LM, which again requires $O(n)$ time. As the whole procedure needs linear time for each $i=1, \ldots, n+n_{u}$ the total running time is $O\left(n^{2}\right)$. 
Putting the intermediate results in Lemmas 3 and 4 together, we conclude:

Theorem 2. The one-edge layout problem can be solved in $O\left(n^{2}\right)$ time.

So far, the algorithm requires $O\left(n^{2}\right)$ space to store the tables $F, C^{\prime}, \mathrm{cr}_{v}$, and $\mathrm{cr}_{u}$. If we are only interested in the minimum number of crossings this can easily be reduced to $O(n)$ space as all tables can be computed row-wise: in $F$ we need only two consecutive rows at a time and we can discard previous rows; in the other tables the rows are independent and can be computed on demand. This does not affect the time complexity. However, to restore the optimal placement we need the pointers in $F$ to do backtracking and hence we cannot easily discard rows of the matrix. But we can still reduce the required space to $O(n)$ with a method similar to a divide-and-conquer version of the Needleman-Wunsch algorithm for biological sequence alignment [2] adding a factor of 2 to the time complexity. Basically, the idea is to keep only the pointers in one column of the matrix to reconstruct the optimal position of the corresponding line. This line cuts the problem into two smaller subproblems which are solved recursively.

\section{Generalization to a Path}

Surely it is desirable to draw lines on a more general fraction of the graph than only on a single edge. However, the problem seems to become significantly harder even for two edges. Let us first define the problem on a path.

\section{Problem 2. Path layout}

Given a graph $G=(V, E)$ and a simple path $P=\left\langle u, w_{1}, \ldots, w_{m}, v\right\rangle$ in $G$. Let $L_{P}$ be the set of lines that use at least one edge in $P$. We split $L_{P}$ into three subgroups: $L_{u v}^{P}$ is the set of lines that have no terminal station in $\left\{u, w_{1}, \ldots, w_{m}, v\right\}$, $L_{u}^{P}$ is the set of lines for which $u$ is an intermediate station and that have a terminal station in $\left\{w_{1}, \ldots, w_{m}, v\right\}$, and $L_{v}^{P}$ is the set of lines for which $v$ is an intermediate station and that have a terminal station in $\left\{u, w_{1}, \ldots, w_{m}\right\}$. We assume that there are no lines having both terminal stations in $\left\{u, w_{1}, \ldots, v\right\}$ as these could be placed top- or bottommost causing the minimum number of crossings with lines in $L_{u v}^{P}$. We also assume that any two lines $\ell_{1}, \ell_{2}$ that use exactly the subpath $x, \ldots, y \subseteq P$ together and for which neither $x$ nor $y$ is a terminal station enter $P$ in $x$ in a predefined order and leave $P$ in $y$ in a predefined order. The task is to find a layout of the lines in $L_{P}$ such that the number of pairs of intersecting lines is minimized.

We tried to apply the same dynamic-programming approach as for the one-edge case. However, the dilemma is that the generalized version of Lemma 1 does not hold, namely that no two lines in $L_{v}^{P}$ intersect. Thus, the problem instance cannot be separated into two independent subproblems, which seems to forbid dynamic programming. In Figure 10 (a) we give an instance where two lines of $L_{v}^{P}$ cross in the optimal solution. Here, the lines in $L_{u v}^{P}$ are drawn solid. Recall that we do not have to take the intersections of lines in $L_{u v}^{P}$ into account as 


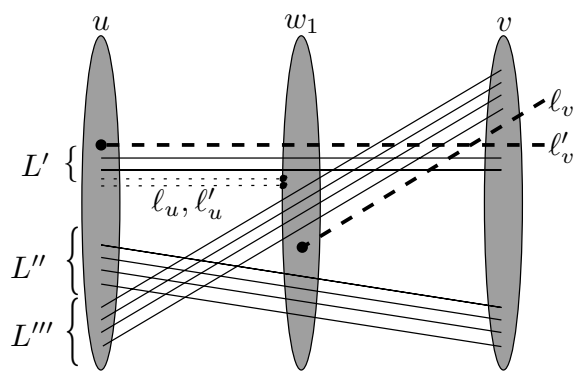

(a) Optimal solution (7 crossings).

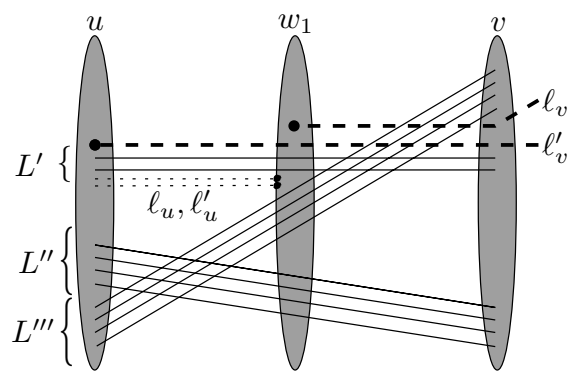

(b) Manipulating $\ell_{v}$ (8 crossings).

Fig. 10. The two lines $\ell_{v}, \ell_{v}^{\prime} \in L_{v}^{P}$ cross in the optimal solution

these are again given by the orders in $S_{u}$ and $S_{v}$. The two lines $\ell_{u}$ and $\ell_{u}^{\prime} \subseteq L_{u}^{P}$ are only needed to force the bundle crossings between the bundles $L^{\prime} \subset L_{u v}^{P}$ and $L^{\prime \prime \prime} \subset L_{u v}^{P}$ to be on the edge $\left\{w_{1}, v\right\}$. In the optimal solution the lines $\ell_{v}, \ell_{v}^{\prime} \in L_{v}^{P}$ intersect causing a total number of 7 crossings between lines in $L_{v}^{P}$ with lines in $L_{v}^{P} \cup L_{u v}^{P}$. We have to argue that any solution in which $\ell_{v}$ and $\ell_{v}^{\prime}$ do not cross produces more than 7 crossings. We look at the optimal solution and argue that getting rid of the crossing between $\ell_{v}$ and $\ell_{v}^{\prime}$ by manipulating the course of either $\ell_{v}$ or $\ell_{v}^{\prime}$ produces at least 8 crossings. First, we consider manipulating the course of $\ell_{v}$, see Figure 10(b). However then, as $\ell_{v}$ has to be above $\ell_{v}^{\prime}$, it has to cross the 4 lines in the bundle $L^{\prime \prime \prime}$ resulting in a total number of 8 crossings. Similarly, manipulating the course of $\ell_{v}^{\prime}$ would also result in at least 8 crossings.

\section{Concluding Remarks}

Clearly our work is only a first step in exploring the layout of lines in graphs. What is the complexity of the problem if two edges of the underlying graph are considered, what about longer paths, trees and finally, general plane graphs? A variant of the problem where lines must terminate bottom- or topmost in their terminal stations is also interesting. This requirement prevents gaps in the course of continuing lines.

\section{References}

1. P. F. Cortese, G. D. Battista, M. Patrignani, and M. Pizzonia. On embedding a cycle in a plane graph. In P. Healy and N. S. Nikolov, editors, Proc. 13th Int. Symp. Graph Drawing (GD'05), volume 3843 of LNCS, pages 49-60. Springer-Verlag, 2006.

2. R. Durbin, S. Eddy, A. Krogh, and G. Mitchison. Biological Sequence Analysis. Cambridge University Press, 1998.

3. M. R. Garey and D. S. Johnson. Crossing number is NP-complete. SIAM J. Alg. Disc. Meth., 4:312-316, 1983.

4. M. Nöllenburg and A. Wolff. A mixed-integer program for drawing high-quality metro maps. In P. Healy and N. S. Nikolov, editors, Proc. 13th Int. Symp. Graph Drawing (GD'05), volume 3843 of LNCS, pages 321-333. Springer-Verlag, 2006. 